screaming with pains in head and neck. Any movement, even stvallowing, increased the pain.

She was admitted late on July 21st, and when seen next day she lay on her right side with legs drawn up and head retracted the meck muscles were rictid , she was conscious but drowsy, cuite intelligent, occosionally screaming out with poin. 'There Taite intelligent, occast vas no paralysis, but she not attempt to move herself. Temperature $103^{\circ}$, respirations 28, palse 108, feeble. Bowels constipated since initial diarrhoex. Throat healthy, ear normal, no herpes, no purpuric spots. Teeth very carions, apex. Abdomen not retracted; no ascites; liver and spleen normal. The urine contained albumin. No muscular tenderuess or wasting; Kernig's sign not present. Knee-jerks absent, abdominal reflexes present; plantar, left extensor, right flexor. Oppenheim's reflex well marked. Pupils small, equal, reacted well. No squint. No nystagmus. Optic disc normal. On lumbar puncture $20 \mathrm{c.cm}$. of cerebro-spinal Huid cripped out slowly; it contained albumin, a moderate number of cells, chiefly polymorphonuclears, and abundant pneumococci in pure culture. Urotropin gr. iij was given every hour.

On July 23 rd she had not vomited since admission; she was delirious, and talked incessantly when awake. On July 24th there was incontinence of urine, the pulse was more feeble, and her general condition worse. She was more drowsy. At 11 p.m., under an anaesthetic, 7 c.cm. antipneumococcic serum was injected into the spinal canal, and on the 24 th another $5 \mathrm{c.cm}$. ; injected into the spinal canal, and on the 24th another 5 c.cm. ; the latter dose was followed by a prolonged rigor some hour ater, and the temperature rose to $106.8^{\circ}$, falling rapidly to $103^{\circ}$ on sponging with cold water. On the 25th she was drowsy and delirious, pulse 160, running; breathing laboured, and pupils dilated. She had emaciated very rapidly since admission.

She became comatose, and died early on July 26 th.

Case virr.-Pneumococcic Meningitis.

A second case of pneumococcic meningitis was admitted in a moribund state on September 19th, 1913, and was of the fulminating form. The patient was a girl, aged 10, who had never had pneumonia or any serious illness, was not deaf nor had any discharge from the ears. After suffering from bod che for the previons two days, she was token suddenly ill with ache for the previous two days, she was taken suddenly ill with high fever, intense headache, and delirium, about 8 p.m. and was brought up to the hospital about 11 p.m. unconcious, with a temperature of $103.5^{\circ}$, a very rapid, feebl dis cerebro-spinal fiuid gave an abundant cellular exudate of poly morphs and lymphocytes, the former being in the proportion of 70 per cent., together with numerous pneumococci.

CASE IX.-Pneumococcic Meningitis.

The third case of pneumococcal infection was in a man of 32 years; he was given 90 c.cm. antipneumococcic serum by intraspinal injection, and recovered after a long convalescence.

REFERENCES.
1 Meningococcus Meningitis, J. B. Lippincott, 1913, page 167. 3 Bitish Medical Jodrnal, 1912, vol. i, January, i913.

\section{A METHOD OF ANAESTHETIZING THE} LARYNX.

By COURTENAY YORKE, M.D.Liverpool, M.B., B.S.Lond., F.R.C.S.Eng.

HONORARY LARYNGOLOGIST TO THE IIVERPOOL HOSPITAL FOR CONSUMPTION $\triangle$ ND DISEASES OF THE CHEST.

Anaesthesia of the laryux is a necessary preliminary in many laryngological procedures, especially in the removal of tumours and in the use of the actual cautery. In the majority of cases the application of cocaine, with brush and syringe, in 10 or 20 per cent. solutions, suffices to render the larynx insensitive. There are, however, not a few patients in whom for various reasons cocaine will not produce the requisite anaesthesia. An inflamed or infiltrated mucous membrane does not respond to cocaine as does a normal healthy one, and it is not surprising, therefore, to find that in tuberculous and other inflammatory states of the larynx anasthesia by the instillation of cocaine is frequently inadequate. Moreover, if it be desired to make a deep puncture with the actual cautery the superficial anaesthesia produced by cocaine is often insufficient. And again there are larynges, not necessarily inflamed, but so irritable that they cannot with cocaine be brought to that complete insensibility which is so essential for exact manipulation. Not infrequently I have, over a period of half an hour or more, applied as much as 10 to 15 grains of cocaine to the larynx without obtaining full anaesthesia, and finally have had to desist from fear of serious toxic symptoms. It is of course understood that in such cases the palate, fauces, pharynx, and base of the tongue were anaesthetized at the outset, while most of the patients had received an injection of morphine and atropine.

For some years the practice has been in rogue of relieving the pain in tuberculous laryngitis by injections of alcohol around the internal laryngeal nerves. Considerable success has attended this procedure, the pain becoming abated for a time, varying from several days to several months. It occurred to me that, as the sensory nerves of the larynx were so accessible, it might be a useful device, in very inflamed and irritable conditions, to inject a solution of novocain around these nerves, and thereby induco anaesthesia of the larynx. This idea, I have recently ascertained, is not original. It was proposed by Frey as long ago as 1906, but so far as I am aware it has not yet become adopted by laryngologists. For the past two years, at the Liverpool Consumption Hospital, I have been practising this method in suitable cases. In all I liave so treated 55 patients, and while it is true that success was not invariable in the earlier cases, with the improvement of the technique my results have become uniformly satis. factory. A preliminary injection of morphine and atropine is advisable, and it is of course necessary to apply cocaine to the palate, fauces, pharynx, base of the tongue; and epiglottis (the last-named structure is supplied by the glossopharyngeal nerve, as well as by the internal laryngeal). In cases in which the palate was extremely sensitive $I$ have occasionally, with great advantage, injected novocain in the neighbourhood of the posterior palatine foramina, and several times I have been obliged similarly to anaesthetize the epiglottis and base of the tongue, puncturing just above the hyoid. The 55 cases referred to included 9 with new growths of the larynx, 2 with singers' nodes, and 1 with lupus. The remainder of the patients suffered from tuberculous laryngitis requiring the use of the actual cautery.

If, after injection of novocain around the nerves, the larynx is still sensitive, the technique has been at fault. As a rule, in such cases, the conductivity of the nerves is interfered with, but not completely blocked, as shown by the fact that a small application of cocaine will now

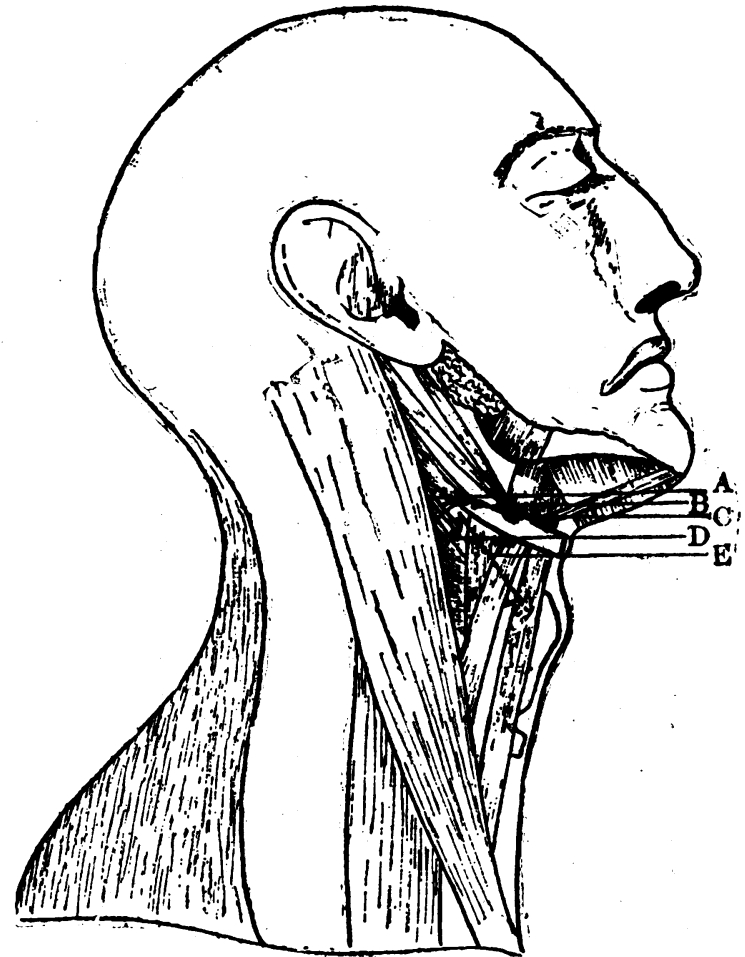

Fig. 1.-A, Internal laryngeal nerve: $B$, point where needle strikes the great cornu of the hyoid bone; c, small cornu of hyoid bone; $D$, bare area of the thyro-hyoid me
followed by the needle during injection.

quickly induce full anaesthesia. The branches of the internal laryngeal which are most likely to escape the novocain are those which supply the arytenoid cartilage and upper part of the epiglottis respectively. I wish particularly to emphasize the fact that novocain injection, even when not fully adoquate, is always a valuable adjunct to cocaine, considerably reducing the amount of 
that drug required. I can affirm most definitely that with the combination of novocain injection and cocaine instil. lation I have never met with a larynx which could not be rendered absolutely insensitive.

Several methods have been described for reaching the laryngeal nerves with the hypodermic needle. I have not found these satisfactory, and their unreliability is apparent,

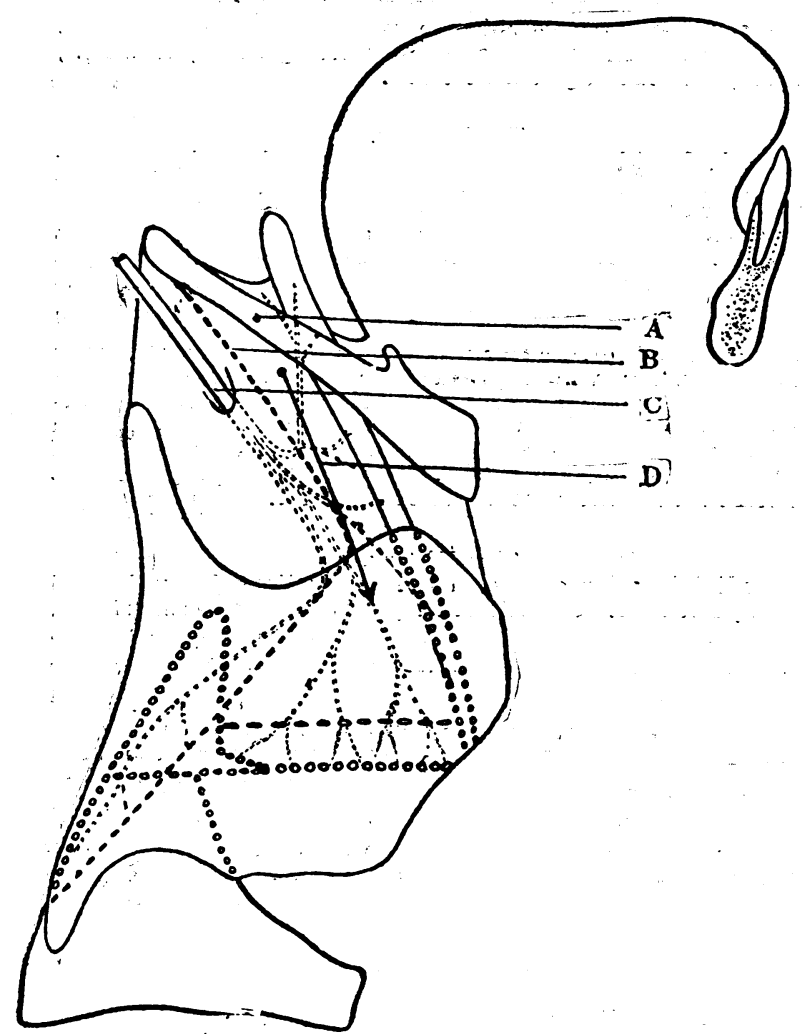

Fig. 2.-A, Point where needle strikes the great cornu of the hyoid bone; B, line indicating anterior limit of the sinus pyriformis: C, internal laryngeal nerve; $D$, line followed by the needle (represented rather far forward).

I think, from the great variability in the results obtained with alcoholic injections by different clinicians. With the object of improving the technique I have made post. mortem dissections on ten bodies, and have examined a large number of anatomical specimens. From the information thus obtained $I$ am able to propose a new and, I believe, much more efficient technique.

All previous methods of injecting the internal laryngeal nerve have aimed at.reaching the nerve as it lies on the thyro-hyoid membrane just before piercing that structure. For anatomical reasons such an attempt is likely to prove very uncertain, for in this position the nerve is deeply placed beneath the sterno-mastoid, while it is only separated from the pharynx by the thyro-hyoid membrane and mucous membrane. The needle being thrust in at right angles to the surface the chance of just striking the plane of the nerve, neither going too deeply nor yet too superficially, is small ; while the necessity for directing the needle so far backwards places the great vessels in danger.

In the method which I have devised the needle is directed along the course of the nerve from the point where it pierces the thyro-hyoid membrane to a point half an inch or so below the upper border of the thyroid cartilage. The solution is injected during the progress of the needie, and in this way one inch or more of the nerve, in a position easily accessible, is brought under the effect of the novocain. The first desideratum, however, in this technique is to get the point of the needle into the plane of the nerve. With this in view the needle is entered so. as to strike the great cornu of the hyoid (a landmark easily felt) one inch behind the lesser cornu. The point is now slightly depressed, so that it hitches against the lower border of the great cornu. The needle is now in relation to the uncovered area of the thyro:hyoid membrane (Fig. 1), and in a position from which to commence the downward and forward movement along the nerve.

The progress of the needle beneath the thyroid cartilage must have regard to the sinus pyriformis. The anterior limit of the pharynx is variable, and dependent on the size of the thyroid cartilage. I have found that, as a rule, in the male the sinus pyriformis, at the upper border of the thyroid, is one inch to one inch and a quarter, and in the female three-quarters of an inch to one inch, from the middle line of the neck. To be quite sure that the needle is engaging the tissues of the larynx, and not entering the pharynx, it is necessayy to keep just within these measure. ments. There is very little likelihood of the needle enter. ing the pharynx in the eavlier part of its course, as it is proceeding in a plane parallel with the thyro-hyoid mom. brane. It only begins to pierce the tissues deeply when it sinks beneath the upper border of the thyroid, and hence the importance, in this latter position, of keeping in front of the sinus pyriformis. In Fig. 2 I have represented the anterior limit of the pharynx by a thick dotted line; while the course of the needle is indicated by an arrow. The diagram illustrates how the branch to the arytenoid may escape; and it will be seen how an earlier origin of the twig to the epiglottis and pharyngeo-epiglottic fold will sometimes place the latter branch also beyond the reach of the novocain. Fig. 3 shows the relation of the internal laryngeal nerve to the sinus pyriformis. It will be seen that for nearly one inch the nerve is only separated from the cavity of the pharynx by mucous membrane.

I do not, when injecting, attempt to elicit pain in the ear, said to indicate contact with the nerve. In my experience, such pain is diffictlt to elicit, most unpleasant to the patient, and not reliable as a guide to the nerve.

During the injection the patient lies in the recumbent position, with the neck well extended, so as to open up the thyro-hyoid interval. A strong sharply-pointed noedle, two inches and a half long, is employed, and the fluid used is a 5 per cent. aqueous solution of novocain, to which a small quantity of adrenalin is added; 20 to $30 \mathrm{~m}$. of this solution are injected into both sides." Weaker solutions of novocain may be used in certain cases, but the stronger solution is quicker and more sure in action. I have never known toxic symptoms ensue with the use of novocain, and the injection method is for this reason specially useful in patients possessing an idiosyncrasy towards cocaine. On several occasions I have tried urea and quinine hydrochloride, but the results were disappointing.

In the 55 cases injected in the manner described, I have never encountered either haemorrhage or inflammatory reaction. The large vessels, if pressed back with the

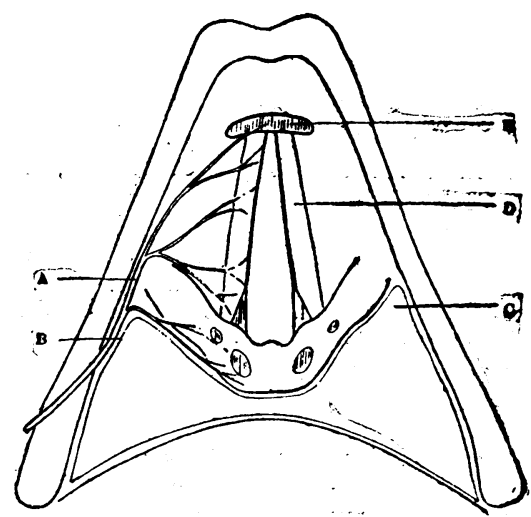

Fig. 3.-A, Internal laryngeal nerve: B, mucous membrane of the pharynx; C, sinus pyriformis ; D, vocal corcous membrane

thumb, are quite out of danger, and, with aseptic precautions, the risk of infection is insignificant.

Summary.

Anaesthesia of the larynx by novocain injections around the internal laryngeal nerves is of value: $(a)$ When cocaine will not induce complete anaesthesia, as in inflammatory and highly irritable conditions of the larynx; $(b)$ when deep anaesthesia is required, as when 'using the actual cautery; $(c)$ when the patient is unduly sensitive to the toxic properties of cocaine. 